\title{
Estimation of Intimacy Change in Team Using Vital Signs
}

\author{
Yuto Hattori, Tomoki Tanaka \\ Graduate School of Science and \\ Engineering Ritsumeikan University, \\ Shiga, Japan \\ Email:\{yuto.hattori, tomoki\} \\ @ de.is.ritsumei.ac.jp
}

\author{
Yusuke Kajiwara \\ College of Production System Engineering \\ and Science Komatsu University, \\ Ishikawa, Japan \\ Email:yusuke.kajiwara@komatsu-u.ac.jp
}

\author{
Hiromitsu Shimakawa \\ College of Information Science and \\ Engineering Ritsumeikan University, \\ Shiga, Japan \\ Email:simakawa@cs.ritsumei.ac.jp
}

\begin{abstract}
In this research, we propose a method using vital signs, to estimate changes of the intimacy inside a team from interactions of team members in the same space. The method estimates both intimacy change between two members and that among whole members. The method facilitates team leaders to grasp the relationships and improve the team performance. Since various measurements representing features of the pulse wave are known to reflect personal emotion, we can expect to estimate the change of intimacy, providing the measurements with a machine learning algorithm. An experiment evaluating the proposed method showed high accuracy in the estimation among all members, but low accuracy in the estimation between two members. In both cases, the accuracy can be improved by choice of effective measurements. Through this experiment, we have found it is necessary to decide the effective measurements for each team to construct a model to estimate intimacy inside the team.
\end{abstract}

\section{INTRODUCTION}

T

EAMS AIM to achieve common goals and objectives through information sharing and mutual support among interaction with other members. Team works can bring higher performance than personal efforts. However, there is a possibility to drop in performance of the team, because relationships between members get worse, or opinions are clashed because of differences in their thoughts and positions. To prevent and solve this problem, team leaders need to grasp relationships of team members at an early stage. If they succeed in it, they can improve the team performance [1].

However, teams consisting of many people form a number of relationships. The relationships change depending on the interaction between members. It is difficult for a team leader to grasp all relationships, which affects the team performance. Existing researches on human relationships analyze information exchanged among remote persons [2]-[6]. However, these tools may fail to reflect the actual interpersonal relationships. It is also suggested there may be privacy concerns in the analysis. In contrast, researches on human relationships in one space analyze conversation information transmitted to other people, but it is difficult to acquire the information in a real time manner. The record of the conversation might prevent team members from interacting as usual.

This paper proposes a method to estimate the change of the intimacy, which is an essential aspect of human relationships.
It examines two aspects: between two members and among whole members. For the estimation, we focus on an individual emotional state rather than information exchanged with others, because intimacy develops through arousing emotions. The pulse wave of each member is acquired by sensors in the course of team member interaction. Various feature quantities are calculated from the pulse wave indicating the emotional arousal. We estimate the change of the intimacy, providing the feature quantities with a machine learning algorithm. The estimated result makes it easy for team leaders to grasp relationships, which enable them to improve the team performance at an early stage.

\section{ESTIMATION OF HUMAN RELATIONSHIPS WITH VITAL SIGNS}

\section{A. Estimation of human relationships}

Existing research on estimation of human relationships have mainly used information in communication. Lin estimated relationships from the frequency of e-mails and mailing lists [2]. Garcia and Vigas et al. proposed a system which visualizes the number of exchanged messages and words that characterize one's correspondence with individuals, to estimate how the relationships have changed over time [3] [4]. Tago and $\mathrm{He}$ et al. used information in social media services to study of relationships [5] [6]. The method engages in estimating relationships in communication of people in different spaces. It is not available to estimate the relationships among people in one space such as a meeting.

Nishihara et al. examine the text of utterances between two persons [1]. They identify the role of the text from the combination of the particle and auxiliary verb in order to estimate the relationships. However, it costs high and consumes a lot of time to create the text of utterances that has been exchanged in a place such as a meeting. Furthermore, it is difficult to estimate the specific relationship when there are multiple relationships.

Many phonetic researches use the change of prosodic information such as pitch (fundamental frequency), speech rate and power [7]-[9]. However, it is difficult to acquire individual prosodic information in the place that many people get together. 


\section{B. The change of the personal emotion in interaction with many people}

Though numerous works have studied detecting emotion in interaction with many people, there are few studies that have focused on human relationship. Hayamizu et al. acquired the facial expressions of group members with cameras, to estimate their emotions [9]. However, the devices which acquire feature information might prevent members from interacting as usual. Ohmoto et al. detected a social atmosphere of extrinsic involvement, enjoyment, or excitement from a body movement and vital signs with the aim to interact as usual [10]. However, it has been shown that expression of body movement varies with individuals in terms of degree of interaction, positions, and personality of individuals. It makes difficult to uniquely determine what body movement express. In addition, it has been shown that it is necessary to use vital signs, because expression of emotion is small when influenced by atmosphere.

\section{Intimacy and emotion}

A degree of intimacy affects teamwork such as communication and cooperativeness. Since individuals appropriately behave to maintain a stable relationship, the paper regards the intimacy as the quality of interactions between people [11] Furthermore, the intimacy develops through the process where individuals disclose their personal information to others [12]. This personal information includes more thoughts or feelings rather than factual information. We focus on emotion in order to estimate the intimacy.

A degree of the intimacy increases, when emotions are transmitted through interaction. Besides, building of the intimacy relationship is promoted through arouses of emotions [13] [14]. Grasp of the change of emotional states would make it possible to estimate the change of the intimacy. Vital signs are often used to estimate the emotional state, because the change of emotional states would often appear on vital signs such as the pulse wave, the blood pressure, and the breathing pace.

\section{The vital signs and emotion}

In order to estimate the emotional state, HRV (Heart Rate Variability) is often used in the vital signs. Since HRV reflects variations in the balance of the sympathetic and the parasympathetic nerve that form autonomic nervous system, it is considered to reflect emotional state [15].

HRV is generally measured by an electrocardiogram. However, it has a disadvantage that its attachment restricts movement of a person. The existing researches suggested photoplethysmography as an alternative approach [16] [17]. It measures the fluctuation of the blood flow in the artery and the capillary by contraction of the heart. Eventually, it measures the pulse wave accompanying the heart rate. Peripheral sites densely packed with capillaries beneath the skin such as fingertips and earlobes are high in detection level. They are suitable to measure the pulse wave. The pulse wave sensor attached to the fingertip is detrimental to actions of a person wearing it, but earlobe causes no problem.

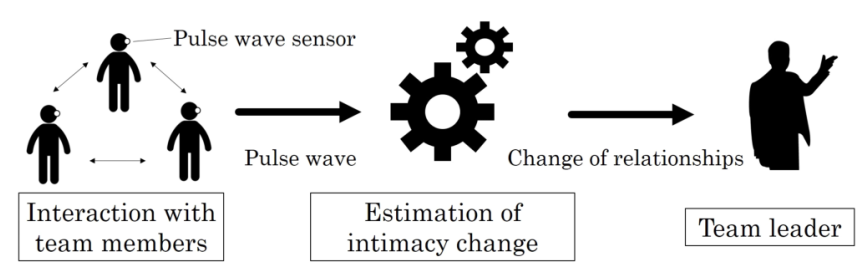

Fig. 1. Use case of method to estimate intimacy change

HR (Heart Rate) and RRI (R-R Interval), which is an index of HRV, are calculated from acquired pulse wave measurements. Activities of the sympathetic nerve brought by arousing emotions such as surprise and pleasure increase HR, while shorten RRI. In contrast, emotions such as relax make parasympathetic nerve active, which decreases HR and makes RRI longer. Furthermore, frequency analysis of RRI figures out the HF (High Frequency) part, and the LF (Low Frequency) part of it. The former ranges from 0.15 to 0.40 $\mathrm{Hz}$, while the latter from 0.04 to $0.15 \mathrm{~Hz}$. The $\mathrm{HF}$ part is used to quantify the parasympathetic nerve fluctuation, while the LF part indicates sympathetic and parasympathetic nerve fluctuation. Thus, the sympathetic nerve fluctuation is examined with LF/HF. HFnorm, which expresses the rate of HF components could be more powerful to reflect the sympathetic nerve fluctuation than HF [18]. These feature quantities are effective for estimating the change of the intimacy.

\section{ESTIMATION OF INTIMACY CHANGE WITH VITAL SIGNS}

\section{A. Estimation method of the intimacy change}

In this research, we propose the method using the pulse wave that is one of the vital signs, to estimate the change of intimacy inside a team. It is acquired with a pulse wave sensor attached on an earlobe of each team member. The method detects activities of the autonomic nerve. The more frequently the emotions are aroused, the larger the change in the activity of the sympathetic and the parasympathetic nerve configuring the autonomic nerve. When the degree of intimacy increases, the frequency of arousing emotions between team members is high, and change in activities of the autonomic nerves is large. On the contrary, when it decreases or does not changes, its activities hardly change. Therefore, the intimacy change of team members can be estimated from activities of the autonomic nerves.

Fig. 1. shows a use case of the method to estimate intimacy from the vital sign. Most of existing researches on human relationships focuses on those between the two people, but few researches focus on those among the whole team. In this research, we estimate not only the change of intimacy between two members but also that inside a team. The leader of team members can easily grasp the relationships among them from the initial stage after the team formation, using the estimation method. The method makes him improve the performance of the team. 


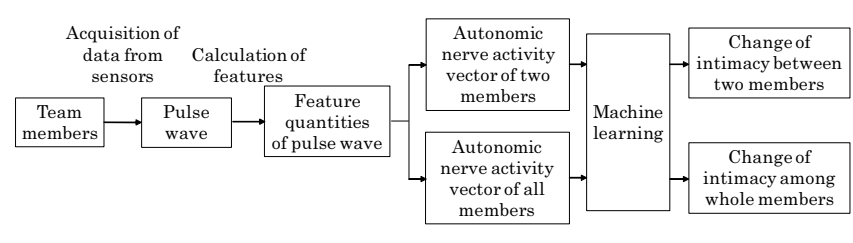

Fig. 2. The flow of the method to estimate change of intimacy

\section{B. Construction of estimation model}

Fig. 2. shows the flow of the method to estimate each change of intimacy. Pulse wave is measured using a sensor attached to each member during they join to an event. The method regards the event as a period of a team activity where they interact with each other. The pulse wave of each member is analyzed to figure out the feature quantities described in II.D. Although a member influences other member, the autonomic nervous is not necessarily activated at the same time. We do not take changes in the course of the time into consideration. The activity vector of the autonomic nervous is composed of the mean and standard deviation of each feature quantities of a member $i$ in the team is $\boldsymbol{p}_{\boldsymbol{i}}$. A person who belongs to the team consisting of $n$ members has relationships with $n-1$ persons, where $n>2$. It is not clear which member affects the change of the autonomic nervous of the person. Therefore, the intimacy change between specified two members $(i, j)$ is estimated by providing $\boldsymbol{q}_{i j}$, the activity vectors of autonomic nervous of the member $i$ and $j$, determined from the following equation (1), to the machine learning algorithm.

$$
\boldsymbol{q}_{i, j}=\boldsymbol{p}_{\boldsymbol{i}}+\boldsymbol{p}_{j}
$$

The intimacy change among whole members is estimated using these activity vectors of autonomic nervous $r$, determined from the following equation (2) to the machine learning algorithm.

$$
\boldsymbol{r}=\sum^{n} \boldsymbol{p}_{\boldsymbol{i}}
$$

We use a supervised learning. To constitute training data, the degree of intimacy evaluation of the other members is acquired two times by the questionnaire: before the event starts and after it ends. The difference these evaluations is regarded as the degree of intimacy change. The intimacy between two members and among whole members is classified based on the difference. This research defines 3 classes to estimate intimacy change: "Good Intimacy", "Constant Intimacy" and "Bad Intimacy", when the degree of the intimacy in questionnaire is increased, unchanged, and decreased, respectively. In the supervised machine learning, these three kinds of intimacy are adopted as objective variables, while the total activities of the autonomic nervous of target members are used as explanatory variables. The proposed method constructs an estimation model of intimacy change with training in the supervised learning.

\section{EXPERIMENT}

\section{A. Experiment overview}

In this experiment, we acquired a pulse wave by the sensor when members are participating in the events. We evaluated possibility to estimate the intimacy change both of between two members and among whole members, using feature quantities acquired from the sensor.

Since the pulse wave differs depending on gender and age, subjects were selected 18 males who are undergraduate and graduate students. They were from 20 to 24 years old; whose average age is 21.4 years old. They are divided into 6 teams, each of which were constituted of 3 members. 3 teams consisted of strangers, while the others are acquaintances. We organized the stranger-teams, because they have no intimacy and tend to appear change of intimacy. In contrast, the acquaintance-teams already have established intimacy.

In each team, the change in the degree of the intimacy and the autonomic nervous is assumed to be different in early stage of human relationships. We explored the differences in each event.

Each subject wore a pulse wave sensor which is "Vital Meter" manufactured by TAOS on the earlobe to acquire the pulse wave.

As shown in Table I, 6 teams worked on preliminary events to acquire the initial intimacy between members. Its content is finding solutions to current affairs through discussion between members. In the preliminary events, we measured the initial degree of the intimacy within the team. Next, they worked on events to increase the degree of the intimacy. The second events are composed of 4 phases (ib1-ib4), which aim to increase the intimacy gradually. We created unique contents while referring to the existing methods [19]. Subjects answered to questionnaire (seven-point answer scale) about their degree of the intimacy with other members after each phase [20].

\section{B. Method of analysis}

First, we analyze the change of subjective evaluation acquired from the questionnaire and feature quantities calculated from the pulse wave in the events. We use each feature quantities standardized by the following equation (3), taking into account that there are differences in feature quantities of each subject.

$$
\begin{gathered}
L_{i}(t)=\frac{\left(g_{i}(t)-\overline{g_{i}}\right)}{\sigma_{i}} \\
\sigma_{i}=\sqrt{\frac{\sum_{t}\left(g_{i}(t)-\overline{g_{i}}\right)^{2}}{N-1}}
\end{gathered}
$$

where $g_{i}(t)$ is a feature quantity in an arbitrary time, and $\overline{g_{i}}$ is the average of each feature quantity of ib1 to ib4.

Next, we evaluate the estimation accuracy of the intimacy, with regard to both of the intimacy change between two members and that among all members, using machine learning. In the case of two members, we compared total evaluation in all the teams with separate evaluation of the stranger-teams 
TABLE I

THE EXPERIMENTAL PROCEDURE

\begin{tabular}{|c|c|}
\hline Phase name (time/minutes) & Content of phase \\
\hline work(10) & Finding solutions to presented assignment with team members \\
\hline ib1 (6) & Self-disclosure to make other members have interest in \\
\hline ib2 (6) & Self-disclosure including game element to break the tension \\
\hline ib3 (6) & Cooperation work to deepen relationships \\
\hline ib4 (6) & Cooperation thinking to deepen relationships \\
\hline
\end{tabular}

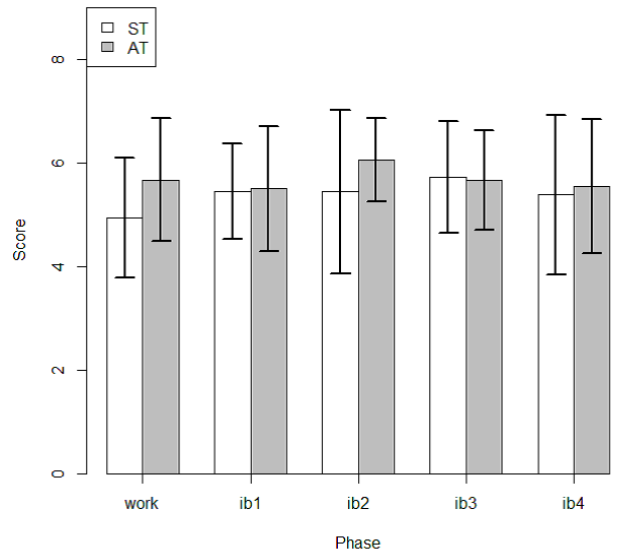

Fig. 3. The mean and standard deviation of questionnaire results

and the acquaintance-teams in the experiment. In case of all three members, we evaluate only total evaluation in all the teams, because the sample size is small.

As a machine learning algorithm, we use the Random Forest, whose inputs are 12 variables composed of the activity vector of two members and all members. In addition, we also tried another estimation using only 3 important variables selected with the Random Forest importance measures. The one team is set test data and the others is training data. We evaluate the accuracy through 6 cross validation in estimation of all teams, while 3 cross validation in stranger-teams and acquaintance-teams. The evaluation index of the accuracy is F-measure determined from equation (5).

$$
F-\text { measure }=\frac{2 \times \text { Precision } \times \text { Recall }}{(\text { Precision }+ \text { Recall })}
$$

\section{Analysis of subjective evaluation}

We analyze the subjective evaluation acquired from the questionnaire as the degree of the intimacy at event participation. Fig. 3. shows the mean and the standard deviation of each stranger-teams(ST) and acquaintance-teams(AT). No significant differences are observed.

Next, we focus on the degree of intimacy changes through each event. The amount of changes in the degree of intimacy with other members is determined from the following equation (6).

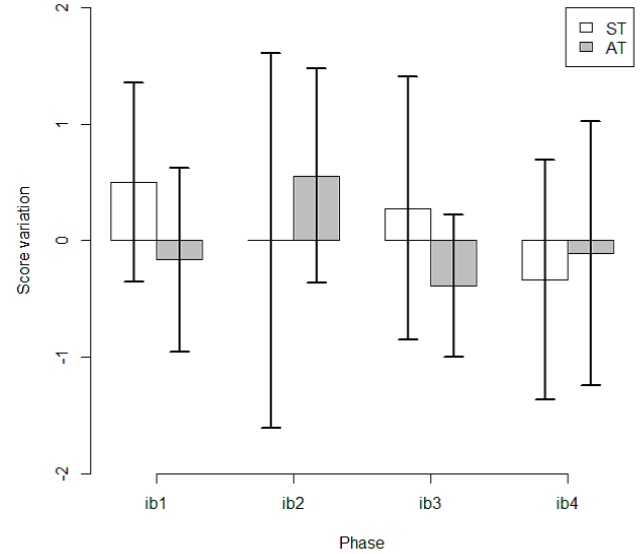

Fig. 4. The mean and standard deviation of the amount of change in the degree of intimacy with another member

$$
\Delta x_{i j, n}=x_{i j, n}-x_{i j, n-1}
$$

where $x_{i j, n}$ represents the result of evaluation for the member $(j)$ by the member $(i)$ after ibn is finished $\left(1 \leq x_{i, j} \leq 7\right)$. $\Delta x_{i j, n}$ is the amount of change in evaluation from the previous event, where $n=0$ means the preliminary work. Fig. 4. shows the mean and the standard deviation of $\Delta x$ in each team.

In stranger-teams, the degree of intimacy increased in ib1 and ib3, whereas decreased in ib4. In acquaintance-teams, the degree of intimacy decreased except in ib2. It represents that strangers have more feeling of closeness through the events. In addition, both of team members do not have a feeling of closeness in the phases but the difference in interaction with other members brings a difference in the degree of intimacy changes.

\section{Analysis of feature quantities}

We analyze the change of each feature quantity calculated from a pulse wave in each phase. The change in the mean of each feature quantity from ib1 to ib4 is calculated by the difference from the preliminary work. Fig. 5. and Fig. 6. show the changes in the mean of HR and RRI which reflect the autonomic nervous fluctuation in each phase of both strangerteams and acquaintance-teams. 


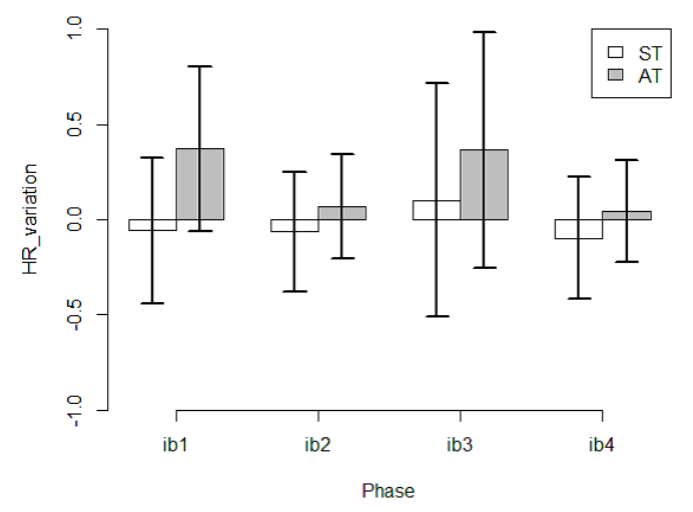

Fig. 5. The change in mean of HR

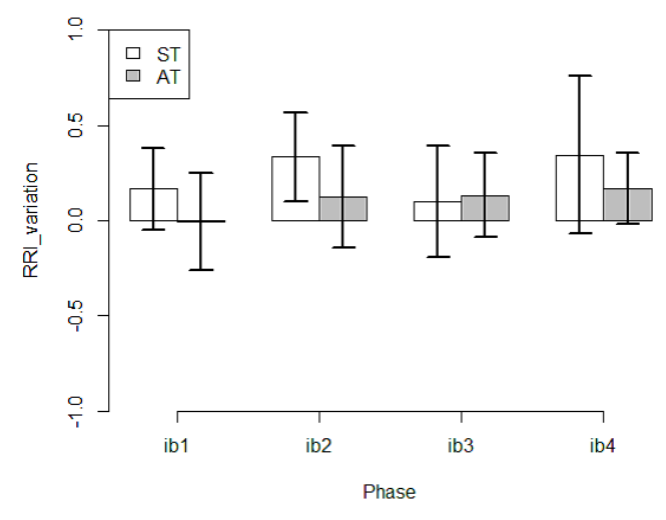

Fig. 6. The change in mean of RRI

In both stranger-teams and acquaintance-teams, the mean of HR was increased in ib3. In the stranger-teams, that in ib1, ib2 and ib4 was decreased. In contrast, in the acquaintance-terms, it was increased in all phases. The mean of RRI was increased except in ib1 of the acquaintance-teams.

Fig. 7. and Fig. 8. show the changes in the mean of HF and HFnorm which reflect the parasympathetic nerve fluctuation.

In both of the stranger-teams and the acquaintance-teams, the mean of HF was decreased from ib1 to ib3. In addition, the mean of HFnorm was decreased in all phases.

Finally, Fig. 9. and Fig. 10. show the changes in the mean of $\mathrm{LF} / \mathrm{HF}$ which reflects sympathetic nerve fluctuation and that of LF which reflects sympathetic nerve and parasympathetic nerve fluctuation, respectively.

In both of the stranger-teams and the acquaintance-teams, the mean of LF/HF was increased from ib1 to ib3. In addition, the mean of LF was increased in all phases.

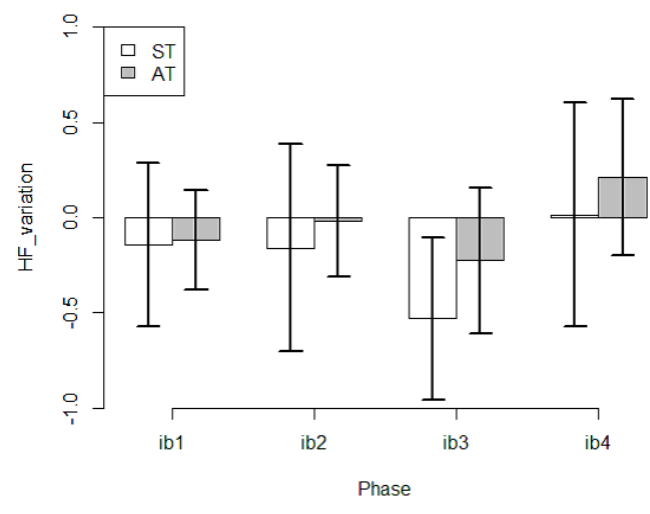

Fig. 7. The change in mean of HF

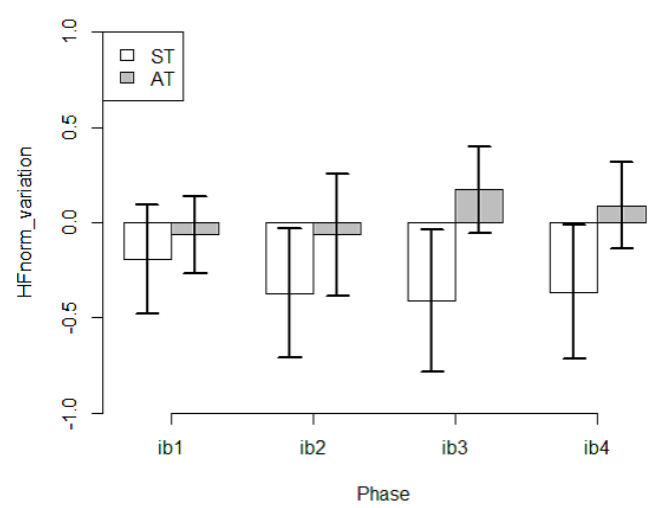

Fig. 8. The change in mean of HFnorm

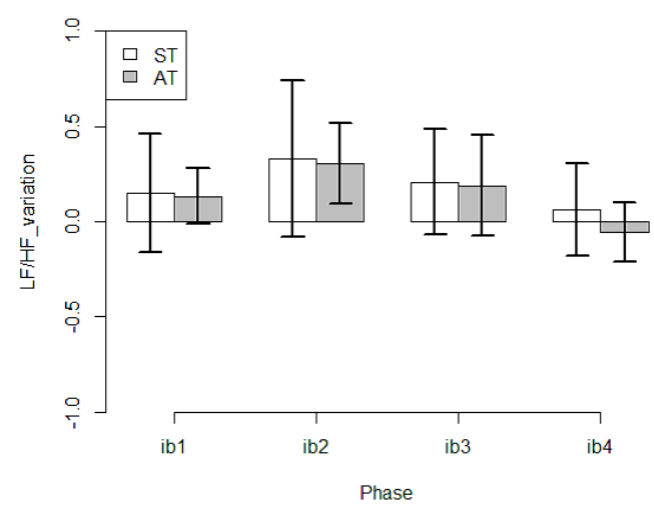

Fig. 9. The change in mean of LF/HF 


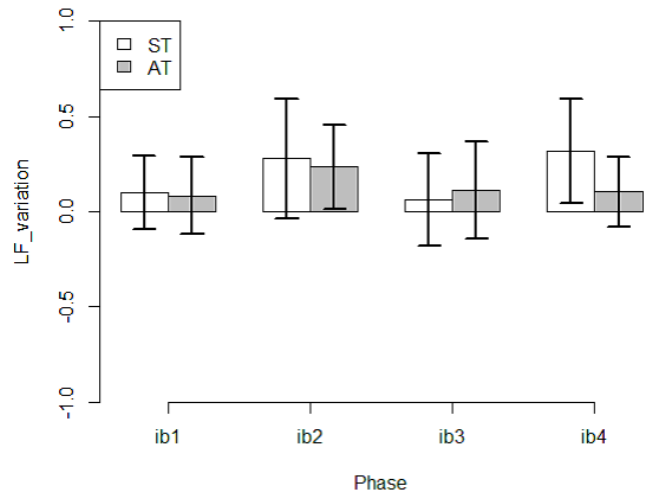

Fig. 10. The change in mean of LF

TABLE II

The SAMPle SIZE OF 3 Classes: "GoOd InTIMACY"(GI), "CONSTANT INTIMACY"(CI) AND "BAD INTIMACY"(BI)

\begin{tabular}{c|c|c|c}
\hline & GI & CI & BI \\
\hline ALL & 24 & 26 & 22 \\
ST & 14 & 13 & 9 \\
AT & 10 & 13 & 13 \\
\hline
\end{tabular}

\section{E. Estimation accuracy of intimacy between two members}

We evaluate the estimation accuracy on three classes of "Good intimacy", "Constant intimacy" and "Bad intimacy" from the changes of the intimacy between two members. The amount of change in the degree of intimacy between two members is determined from the following equation (7).

$$
\Delta y_{i j, n}=x_{i j, n}+x_{j i, n}
$$

where $\Delta y_{i j, n}$ is summing up amount of change from the previous phase in evaluation of members ( $i$ and $j$ ) after ibn is finished. $\Delta y_{i j, n}$ is classified into 3 classes in order to be adapted objective variable: $\Delta y_{i j, n}>0$ ("Good Intimacy"), $\Delta y_{i j, n}=0$ ("Constant Intimacy") and $\Delta y_{i j, n}<0$ ("Bad Intimacy"). Table II shows each sample size.

We estimated using not only both 12 variables, but also 3 variables which is the mean of HR, the mean of RRI, and the standard deviation of RRI as explanatory variable. Fig. 11. shows the estimation accuracy.

The estimation accuracy in case of using 12 variables was low, namely, it is lower than the accuracy of $1 / 3$ which is random guess. In contrast, the estimation accuracy of all teams, stranger-teams and acquaintance-teams in case of using 3 variables was higher than that of 12 variables. The estimation accuracy of the acquaintance-teams was especially high, though that of the stranger-teams was lower than all teams. Table III shows cross-tabulation table in the estimation of all teams. The identification accuracy of "Good Intimacy"

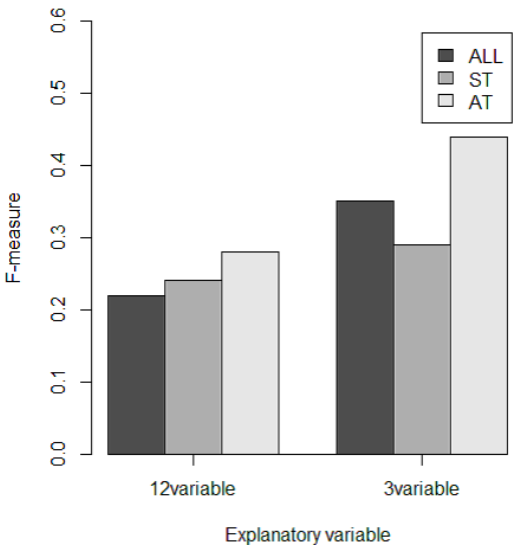

Fig. 11. Each estimation accuracy of intimacy between two members

TABLE III

THE CROSS-TABULATION TABLE IN ESTIMATION OF ALL TEAMS

\begin{tabular}{c|c|c|c}
\hline & BI & CI & GI \\
\hline BI & 278 & 870 & 691 \\
CI & 1186 & 888 & 1208 \\
GI & 736 & 842 & 501 \\
\hline Recall & 0.12 & 0.34 & 0.21 \\
Precision & 0.15 & 0.3 & 0.22 \\
F-measure & 0.13 & 0.3 & 0.22 \\
\hline
\end{tabular}

and "Bad Intimacy" was low. It is misidentified as "Constant Intimacy".

\section{F. Estimation accuracy of intimacy among all members}

We evaluate the estimation accuracy from 3 changes of the intimacy in a team. However, in this case, the sample size was too small to discriminate either of 3 classes or each of stranger-teams and acquaintance-teams. Because of this, we evaluate the estimation accuracy of 2 classes, one of which is "Good Intimacy" and the other is eith of "Constant Intimacy" or "Bad Intimacy" in all teams. The amount of changes in the degree of intimacy among all members is determined from the following equation (8).

$$
\Delta z_{i j k, n}=\Delta y_{i j, n}+\Delta y_{j k, n}+\Delta y_{i k, n}
$$

where $\Delta z_{i j k, n}$ is the sum of the amount of 3 changes from the previous phase in evaluation of members, assuming $\mathrm{k}$ is same team members with $\mathrm{i}$ and $\mathrm{j}$, after ibn is finished. $\Delta z_{i j k, n}$ is classified into 2 classes in order to be adapted objective variable: $\Delta z_{i j k, n}>0$ ("Good Intimacy") and $\Delta z_{i j k, n} \leq 0$ ("Constant Intimacy" and "Bad Intimacy"). The sample size of the two cases is 11 and 13, respectively. We estimated using not only while 12 variables, but also 3 variables which are the mean of HF, the mean of HFnorm and the standard deviation of HFnorm as explanatory variables. The estimation accuracy was 0.69 in case of 12 variable, and 0.76 in 3 variable. In 
estimation among all members, both of the accuracy of 12 variables and 3 variables were high.

\section{DisCUSSION}

\section{A. The change of intimacy and feature quantities in phase}

The stranger-teams were expected that the degree of the intimacy increased in phases through events, while no change was expected in acquaintance-teams. However, the result was quite different; the degree of intimacy changes varies with events. We discuss reasons for this result below.

In ib1, it is assumed that strangers had feeling of closeness by learning about others. In contrast, acquaintances who could obtain only known information from others did not have more than original feeling of closeness. In ib2, acquaintances had feeling of closeness through sharing information while enjoying with others by games. On the other hand, strangers were unaffected with games. Their feeling was unchanged because it was similar to ib1, regarding self-disclosure. In ib3, strangers had feeling of closeness because of close relationships to others through cooperative works. In contrast, acquaintances worked with certain relationships, which made the degree of intimacy decreased from ib2. In ib4, both strangers and acquaintances did not have feeling of closeness because almost everyone concentrated on thinking than discussing among the members.

From HR of the feature quantities calculated from the pulse wave increased in $i b 3$, it is assumed that sympathetic nerve got active, because of aroused emotions such as joy and pleasure coming from increasing opportunities to interact with other members. HR was decreased in ib1, ib2 and ib4 for the stranger-teams. However, HF was decreased in ib1 and ib2. It implies that it might be affected by either tension in works, or temporal change in breathing such as laughters in conversations.

It can be considered that there is a difference in autonomic nervous changes depending on the contents of the interaction. However, the change might vary with person to person. It is necessary to consider that the influence of the sympathetic and parasympathetic nerves on each person.

\section{B. Estimation accuracy of intimacy change}

As a result of estimation based on the features acquired from the pulse wave, it is possible to estimate the intimacy in team. However, estimation between two members is difficult. As the possible reason for this, the interaction of whole team seemed to be more frequent than that between two members. The interaction of whole team affected the emotional arousal of all members. Table IV shows the mean and standard deviation of 3 important variables in each class for estimation of whole team. Only the standard deviation of HFnorm was recognized significant difference in the results of $U$ test whose significance level is $5 \%$. It shows the variance of parasympathetic nerve activity of members who feel close each other is larger than other relationships. In the intimacy between two members, it is assumed that estimation accuracy is low because of many misidentified. It might come from the interaction between two
TABLE IV

THE MEAN AND STANDARD DEVIATION OF 3 VARIABLES IN 2 CLASSES

\begin{tabular}{c|c|c|c|c|c|c}
\hline & \multicolumn{2}{|c|}{ HF_Mean } & \multicolumn{2}{c|}{ HFnorm_Mean } & \multicolumn{2}{c}{ HFnorm_SD } \\
\cline { 2 - 7 } & Mean & SD & Mean & SD & Mean & SD \\
\hline CI,BI & -0.07 & 1.59 & -0.31 & 0.84 & 5.48 & 0.46 \\
GI & 0.09 & 1.03 & 0.36 & 0.9 & 6.17 & 0.44 \\
\hline
\end{tabular}

members. Since it was little, we could not find difference in the activity vectors of autonomic nervous of two members.

In this experiment, since the degree of intimacy was acquired by subjective evaluation, the subjects may evaluate their intimacy increased temporary because of arousing emotions according to the peak-end rule [21]. However, activity vectors of autonomic nervous smooth temporary arousing emotions, which make the estimation accuracy low. The estimation accuracy might improve, if we consider the peak value of each feature quantity and the ratio of the peak over a threshold value in the whole using the Peak-Valley method. The estimation accuracy of both teams was improved by using only 3 important variables. However, the important variables were different in each team. In addition, since a sufficient sample size was not obtained, we cannot decide important feature quantities uniquely, to estimate the intimacy.

\section{ConClusion}

In this paper, we proposed a method to estimate intimacy changes not only between two members but also among whole members using feature quantities calculated from the pulse wave, in order to grasp relationships of team members. From the results of our experiment, the change of intimacy degree was different between strangers and acquaintances, depending on the way of interaction. Individual differences in the effects of autonomic nervous were shown from changes of both parasympathetic and sympathetic. Estimation accuracy of changes in the intimacy among whole members was high enough to estimate relationships. However, the estimation accuracy between two members was low. The selection of only effective feature quantities improved the estimation accuracy in the both cases, but we cannot identify the feature quantities uniquely. It is necessary to select feature quantities for each team. Since the number of samples in this experiment is small, it is necessary to obtain accurate results by condacting an additional experiment and increasing the number of samples in the future.

Since the method examines the pulse wave, it can be applied only in situation where all members stay sitting, such as discussion. In this experiment, we assumed 2 kinds of teams: teams of strangers and teams of acquaintances. However, in actual organizations and companies, there can be teams in which both of them are mixed. It is necessary to examine whether the proposed method works well for such teams in future. 


\section{REFERENCES}

[1] Nishihara, Y., Sunayama, W., \& Yachida, M. (2008). Human Friendship and Hierarchical Relationship Estimation from Utterance Texts, IEICE transactions on information and systems, 91.1: 78-88.

[2] Lin, H. (2010, December). Predicting sensitive relationships from emai corpus. In Genetic and Evolutionary Computing (ICGEC), 2010 Fourth International Conference on (pp. 264-267). IEEE.

[3] Garcia, T., Aires, J., \& Gonalves, D. (2012, May). Who have I been talking to?. In Proceedings of the International Working Conference on Advanced Visual Interfaces (pp. 481-484). ACM.

[4] Vigas, F. B., Golder, S., \& Donath, J. (2006, April). Visualizing email content: portraying relationships from conversational histories. In Proceedings of the SIGCHI conference on Human Factors in computing systems (pp. 979-988). ACM.

[5] Tago, K., \& Jin, Q. (2017, November). Analyzing influence of emotional tweets on user relationships by naive bayes classification and statistical tests. In Service-Oriented Computing and Applications (SOCA), 2017 IEEE 10th International Conference on (pp. 217-222). IEEE.

[6] He, X., Wang, Y., Li, Y., \& Jiang, Y. (2018, January). Investigating Relationships in Online Communities: A Social Network Analysis. In Proceedings of the 2018 2nd International Conference on Management Engineering, Software Engineering and Service Sciences (pp. 229-233). ACM.

[7] Nishimura, R., Kitaoka, N., \& Nakagawa, S. (2008). Analysis of relationship between impression of human-to-human conversations and prosodic change and its modeling. In Ninth Annual Conference of the International Speech Communication Association.

[8] Inaguma, H., Inoue, K. Nakamura, S., Takanashi, K, \& Kawahara, T. (2016, November). Prediction of ice-breaking between participants using prosodic features in the first meeting dialogue. In Proceedings of the 2nd Workshop on Advancements in Social Signal Processing for Multimodal Interaction (pp. 11-15). ACM.

[9] Hayamizu, T., Mutsuo, S., Miyawaki, K., Mori, H., Nishiguchi, S., \& Yamashita, N. (2012, November). Group emotion estimation using Bayesian network based on facial expression and prosodic information. In Control System, Computing and Engineering (ICCSCE), 2012 IEEE International Conference on (pp. 177-182). IEEE

[10] Ohmoto, Y., Miyake, T., \& Nishida, T. (2010). Study on Detecting a Social Atmosphere of Extrinsic "Involvement, Enjoyment, or Excite- ment" in Multi-User Interaction, IEICE transactions on information and systems, 93.6: 870-878

[11] Laurenceau, J. P., Barrett, L. F., \& Pietromonaco, P. R. (1998). Intimacy as an interpersonal process: The importance of self-disclosure, partner disclosure, and perceived partner responsiveness in interpersonal exchanges. Journal of personality and social psychology, 74(5), 1238.

[12] Reis, H. T., \& Shaver, P. (1988). Intimacy as an interpersonal process. Handbook of personal relationships, 24(3), 367-389.

[13] Howell, A., \& Conway, M. (1990). Perceived intimacy of expressed emotion. The Journal of social psychology, 130(4), 467-476.

[14] Graham, S. M., Huang, J. Y., Clark, M. S., \& Helgeson, V. S. (2008) The positives of negative emotions: Willingness to express negative emotions promotes relationships. Personality and Social Psychology Bulletin, 34(3), 394-406.

[15] Task Force of the European Society of Cardiology. (1996). Heart rate variability, standards of measurement, physiological interpretation, and clinical use. circulation, 93, 1043-1065.

[16] Sengthipphany, T., Tretriluxana, S., \& Chitsakul, K. (2015, June). Comparison of Heart Rate statistical parameters from Photoplethysmographic signal in resting and exercise conditions. In Electrical Engineering/Electronics, Computer, Telecommunications and Information Technology (ECTI-CON), 2015 12th International Conference on (pp. 1-5). IEEE

[17] Bolanos, M., Nazeran, H., \& Haltiwanger, E. (2006, August). Comparison of heart rate variability signal features derived from electrocardiography and photoplethysmography in healthy individuals. In Engineering in Medicine and Biology Society, 2006. EMBS'06. 28th Annual International Conference of the IEEE (pp. 4289-4294). IEEE.

[18] Yin, L., Zhang, W., \& Xia, L. (2010, October). Effect of skin pressure caused by the cuff on female heart rate variability. In Biomedical Engineering and Informatics (BMEI), 2010 3rd International Conference on (Vol. 2, pp. 883-886). IEEE.

[19] Hori, K., Kato, A., \& Karube, T. (2007). Team Building: hito to hito wo "tunagu" gihou [Team Building: Technique to connect people]. Tokyo: Nikkei Publishing Inc.

[20] Fujimori, T. (1980). EFFECTS OF ATTITUDE SIMILARITY AND TOPIC IMPORTANCE ON INTERPERSONAL ATTRACTION. The Japanese Journal of Experimental Social Psychology, 20(1), 35-43.

[21] Miron-Shatz, T. (2009). Evaluating multiepisode events: Boundary conditions for the peak-end rule. Emotion, 9(2), 206. 